\title{
Facteurs influençant le choix de la spécialité de médecine générale par les étudiants en médecine
}

\section{Factors influencing student's choice of general practice as a specialty in France}

\author{
Antoine AUBRION ${ }^{1}$, Pascal GONCALVES ${ }^{1}$, Vincent KOWALSKI ${ }^{1}$, Anaïs REICHLING ${ }^{3}$, \\ Vladimir MANSOUR ${ }^{2}$ \\ 1 Département de médecine générale, Faculté de médecine, Université de 14000 Caen, France \\ 2 Service des Urgences, Centre hospitalier de Lisieux, 14100 Lisieux, France \\ 3 Service de Pharmacie, Centre hospitalier universitaire de Caen, 14000 Caen, France
}

Manuscrit soumis pour publication le 5 juin 2015 ; commentaires éditoriaux formulés aux auteurs le 13 mars 2016 ; accepté pour publication le $1^{\mathrm{er}}$ février 2017

\begin{abstract}
Mots-clés
Étudiants en

médecine ;

médecine générale ;

choix de carrière ;

choix professionnel ;

épreuves classantes

nationales ;

démographie

médicale

Résumé - Contexte et problématique : Depuis plus de 20 ans, une diminution du nombre d'étudiants en médecine choisissant de devenir médecin généraliste semble s'observer. Objectif : L'objectif de cette étude était d'étudier les facteurs influençant ce choix chez des étudiants en médecine d'une faculté française. Méthodes : Une étude observationnelle descriptive a été réalisée en mars 2015. Les étudiants de la faculté de médecine de l'Université de Caen (France) inscrits aux épreuves classantes nationales ont été invités à répondre à un questionnaire validé auto-administré divisé en trois parties : renseignements personnels ; choix de spécialité et ville d'affectation ; facteurs influençant le choix de carrière. Les étudiants étaient invités à évaluer dans quelle mesure ils percevaient chacun des 27 items comme influençant leur choix de carrière. Résultats : $77 \%$ de la population était répondante $(n=174)$ dont $46,6 \%$ d'hommes, avec un âge moyen de 25 ans. Un tiers des étudiants s'orientait vers la médecine générale en premier choix. Les facteurs associés au choix de la médecine générale étaient un âge plus élevé et le fait d'avoir effectué un stage en médecine générale pendant le second cycle d'études. Le regroupement des informations en six classes distinctes retrouvait une différence de choix de la médecine générale ou d'autres spécialités selon l'orientation sociétale, les structures de soin, et le prestige attribué. Conclusion : Une préférence des étudiants en médecine pour la médecine générale est associée à une place différente pour trois groupes de valeurs. Ces résultats concordants montrent une certaine adéquation socioculturelle des facteurs d'influence, et leur reproductibilité sur le modèle éducatif médical francophone et français.
\end{abstract}




\begin{abstract}
Keywords
Medical students; family medicine; general practice; career choices; national ranking examinations; medical demography

Abstract - Background : For over 20 years the number of medical students choosing to become general practitioners seems to be declining. Purpose : The purpose of this study was to examine what influences the choice of practice among medical students in a French faculty. Method : A descriptive observational study was conducted in March 2015. The students of the medical faculty of Caen (France) enrolled in the national ranking examinations were asked to complete a validated self-administered questionnaire divided into three sections: personal information; choice of specialty and city of assignment; career-choice influences. The students were asked to rate how they perceived each of the 27 items influencing their career choices. Results : $77 \%$ of the responding group $(n=174)$, which included $46.6 \%$ men, were 25 years old on average. One third of students had selected general practice as their initial choice. The factors associated with choosing general practice were linked to being older and completing an internship in general practice during the second cycle of studies. Information was consolidated into six distinct categories with social trends, hospital structures and prestige making a difference between general practice and all other specialties. Conclusion : Medical students' preference for general practice is associated with a different status for three value groups. These corroborating results indicate some socio-cultural balance in terms of influences and their reproducibility in the French and French-speaking medical education model.
\end{abstract}

\section{Introduction}

\section{Démographie médicale}

Dans la plupart des pays, le nombre et la répartition des médecins sont déterminés par les politiques gouvernementales, dans le cadre de mesures incitatives ou limitantes ${ }^{[1,2]}$. Malgré cela, les choix individuels de carrière ont entraîné un retentissement sur l'offre de soins primaires. Le nombre de médecins par habitant au Canada a ainsi déjà connu une baisse de $5,1 \%$. Aux Etats-Unis une réduction de plus de $20 \%$ de la force de travail en soins primaires est prévue d'ici $2025^{[3,4]}$.

En France, les projections nationales ont prévu une diminution du nombre de généralistes jusqu'en 2019 , avant une reprise progressive permettant un solde entrée-sorties d'activité positif à partir de 2020 et un retour en 2030 au nombre de praticiens en exercice de 2006. La densité des praticiens passerait de 163 à 148 généralistes pour 100000 habitants entre 2006 et 2030, atteignant son point bas en $2022^{[5]}$.

De plus en plus de praticiens choisissent de limiter leur activité en termes d'horaires, par le biais d'une spécialisation, ou en se retirant précocement de leur exercice $^{[6,7]}$. Majoritaires en médecine générale en
France dès 2017, les femmes conduisent ce changement qui souhaite concilier harmonieusement vie professionnelle et vie privée, apportant un certain équilibre aux conditions d'exercice ${ }^{[8,9]}$.

\section{Choix de carrière}

\section{Contexte international}

Au terme de sa formation, l'étudiant en médecine doit se positionner sur le choix d'une spécialité pour l'avenir de sa vie professionnelle. Depuis plus de 20 ans, dans la plupart des pays industrialisés, on observe une diminution constante du nombre d'étudiants en médecine choisissant de devenir médecin généraliste ${ }^{[10-13]}$. La profession n'attire plus assez de candidats. En 2009 au Canada, $32 \%$ des étudiants souhaitaient la médecine générale en premier choix de spécialité contre $20 \%$ en France ${ }^{[14,15]}$.

\section{Contexte des études médicales en France}

En France, au terme des six premières années d'études médicales, les étudiants choisissent leur affectation de spécialité d'internat en fonction de leur rang de classement aux épreuves classantes 
nationales $(\mathrm{ECN})^{[16,17]}$. Le nombre de postes ouverts par les pouvoirs publics détermine chaque année le nombre d'étudiants dans chaque spécialité par subdivision de formation. Après classement, la médecine générale reste la spécialité choisie au second rang chez les femmes et au cinquième rang chez les hommes en $2012^{[18,19]}$. Cependant, certains choix secondaires de réorientation vers des diplômes d'enseignement spécialisés complémentaires ou des diplômes inter-universitaires écartent ces étudiants de leur affectation initiale en médecine générale ambulatoire $(20,5 \% \text { de l'effectif initial })^{[20]}$.

Facteurs d'influence

\section{Études qualitatives}

Face à ces enjeux, la médecine générale est actuellement en questionnement dans la plupart des pays industrialisés ${ }^{[21,22]}$. La compréhension des caractéristiques qui influencent le choix de carrière suscite un grand intérêt et les études proviennent essentiellement du Canada, des Etats-Unis et de pays européens à tradition anglo-saxonne. La plupart des études ont classé les facteurs d'influence selon trois catégories : les caractéristiques socio-démographiques des étudiants, les caractéristiques des facultés, enfin l'interaction entre les caractéristiques des spécialités et les attitudes et valeurs personnelles des étudiants ${ }^{[23-25]}$. D'autres ont étudié leurs traits de personnalité associés au choix de carrière ${ }^{[26]}$. Ces études ont eu recours à des méthodes de recueil d'information de nature qualitative (entretiens semi-dirigés ou groupes de discussion d'étudiants). D'autres se limitaient au recensement de critères sélectionnés par

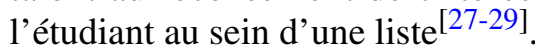

\section{Etudes quantitatives}

Murdoch aux États-Unis en 2001 et Wright au Canada en 2004 ont validé un questionnaire en anglais d'attitudes et de valeurs des étudiants en médecine pour leur choix de carrière médicale future ${ }^{[30,31]}$. Une version française du questionnaire de Wright élaborée par Beaulieu et al. a été validée en 2010 ${ }^{[32]}$.
Jusqu'à présent aucune étude n'a utilisé cet outil sur le territoire Européen.

\section{Objectifs de l'étude}

L'objectif principal de l'étude était d'évaluer les facteurs influençant le choix de la médecine générale chez les étudiants en médecine d'une faculté française, à l'aide du questionnaire de Beaulieu distribué deux mois avant les ECN. Les objectifs secondaires étaient de retrouver des groupes de valeurs statistiquement associées, d'étudier la répartition des choix de ville d'affectations des étudiants, dans chaque spécialité, ainsi que l'influence de l'un sur l'autre.

\section{Méthodes}

\section{Procédure de l'étude}

En mars 2015, une enquête a été menée auprès de tous les étudiants de sixième année de la faculté de médecine de Caen (France) inscrits aux ECN en 2015. Lors d'une épreuve de préparation aux ECN, les étudiants ont été invités à répondre à un questionnaire auto-administré. L'étude a été expliquée aux étudiants par une personne impartiale qui n'avait aucune affiliation avec les étudiants. Les personnes absentes lors de cette épreuve étaient invitées à répondre au questionnaire dans les mêmes conditions lors d'un cours obligatoire la semaine suivante.

\section{Questionnaire}

Le questionnaire est divisé en trois parties (Annexe 1) : critères socio-démographiques ; choix de spécialité et ville d'affectation ; facteurs influençant le choix de carrière. Les étudiants étaient invités à évaluer dans quelle mesure ils percevaient chacun des 27 items comme influençant leur choix de carrière au moyen d'une échelle de Likert allant de 1 (aucune influence) à 5 (grande influence). Ces items étaient regroupés en quatre classes définies par l'étude de Beaulieu ${ }^{[32]}$ et reprises intégralement : style de vie 
médicale, orientation sociétale, prestige, structure de soins. Deux classes supplémentaires étudiaient l'influence de la formation, et du choix aux ECN.

Quatre items ont été ajoutés devant les particularités françaises d'affectation des étudiants, couplant choix de spécialité et du lieu d'affectation selon le rang de classement aux ECN. Ces quatre items étaient la prépondérance du choix de la spécialité sur la ville (ou inversement), l'influence du conjoint et la difficulté de préparation aux ECN. La présence d'un stage de médecine générale ambulatoire dans le cursus de l'étudiant, l'identification à un médecin de cette spécialité, ainsi que l'influence globale des stages ont également été recherchés. La première version du questionnaire a préalablement été testée sur 10 étudiants de l'année inférieure pour éviter toute influence sur les réponses.

\section{Analyse des données}

L'analyse des données a été effectuée en utilisant le logiciel SPSS version 22.0. Le regroupement des facteurs en six classes a été soumis à un test de corrélation de chaque facteur à sa classe. Les variables quantitatives ne décrivant pas une distribution normale, les données ont été interprétées par tests non paramétriques. Les données qualitatives ont été comparées par test de Khi-deux. Les données ont été analysées en mode multivarié entre les populations ayant choisi la spécialité de médecine générale contre ceux ayant choisi une autre spécialité, puis entre les étudiants ayant choisi de conserver le même centre de formation contre ceux ayant choisi une autre ville d'affectation. Un seuil d'erreur de $5 \%$ était défini comme acceptable.

\section{Résultats}

Sur les 226 étudiants de notre population, 176 étudiants ont répondu au questionnaire $(78 \%)$. Deux questionnaires ont été exclus pour absence de réponse au choix de spécialité (Figure 1).
Analyse descriptive

La population étudiée comportait 46,6\% d'hommes ; l'âge moyen était de 25 ans. Parmi les étudiants, 54,6\% étaient en couple et $37.8 \%$ avaient au moins un parent exerçant dans le milieu médical. Un stage en médecine générale ambulatoire pendant leur deuxième cycle d'études avait été effectué par 34,7 \% des étudiants. Cinquante huit étudiants $(33,3 \%)$ s'orientaient vers la médecine générale en premier choix. Les autres étudiants s'orientaient principalement vers l'anesthésie réanimation $(n=21)$, la chirurgie générale $(n=11)$, la pédiatrie $(n=9)$, l'oncologie $(n=7)$ ou la néphrologie $(n=7)$. Parmi ces 116 étudiants ayant choisi une autre spécialité en premier choix, $29(25,0 \%)$ s'orientaient vers la médecine générale en second choix.

Les facteurs ayant le plus d'influence étaient la pratique d'une activité extramédicale (note moyenne de 3,8 sur 5) et la gestion d'un large éventail de problèmes $(3,9)$. Concernant les particularités du cursus, les facteurs les plus influents étaient le stage d'externat $(3,9)$ et l'attachement au choix de spécialité avant celui de la ville $(4,0)$. Chacun des items était corrélé à sa classe, mis à part la classe « influence du choix $\mathrm{ECN} »($ tableau I).

\section{Analyse multivariée : choix de la médecine générale}

Un âge plus élevé et le fait d'avoir effectué un stage en médecine générale ambulatoire pendant le second cycle d'études étaient associés au choix de la médecine générale ( $p=0,04$ et $p=0,02$; tableau II). Les principales villes d'affectation souhaitées étaient situées sur le littoral ouest entre Rouen et Bordeaux, les étudiants s'orientant vers la médecine générale étant moins enclins à l'éloignement que les autres.

Aucune des variables ne répondait à une distribution normale. Les facteurs associés au choix de la médecine générale (tableau III) étaient les possibilités de choix et d'ouverture de leur pratique $(p=0,02$ et $p=0,001)$, la concentration sur une communauté $(p=$ $0,009)$, les relations à long terme avec les patients $(p<$ $0,001)$, l'engagement social personnel $(p=0,01)$, la 


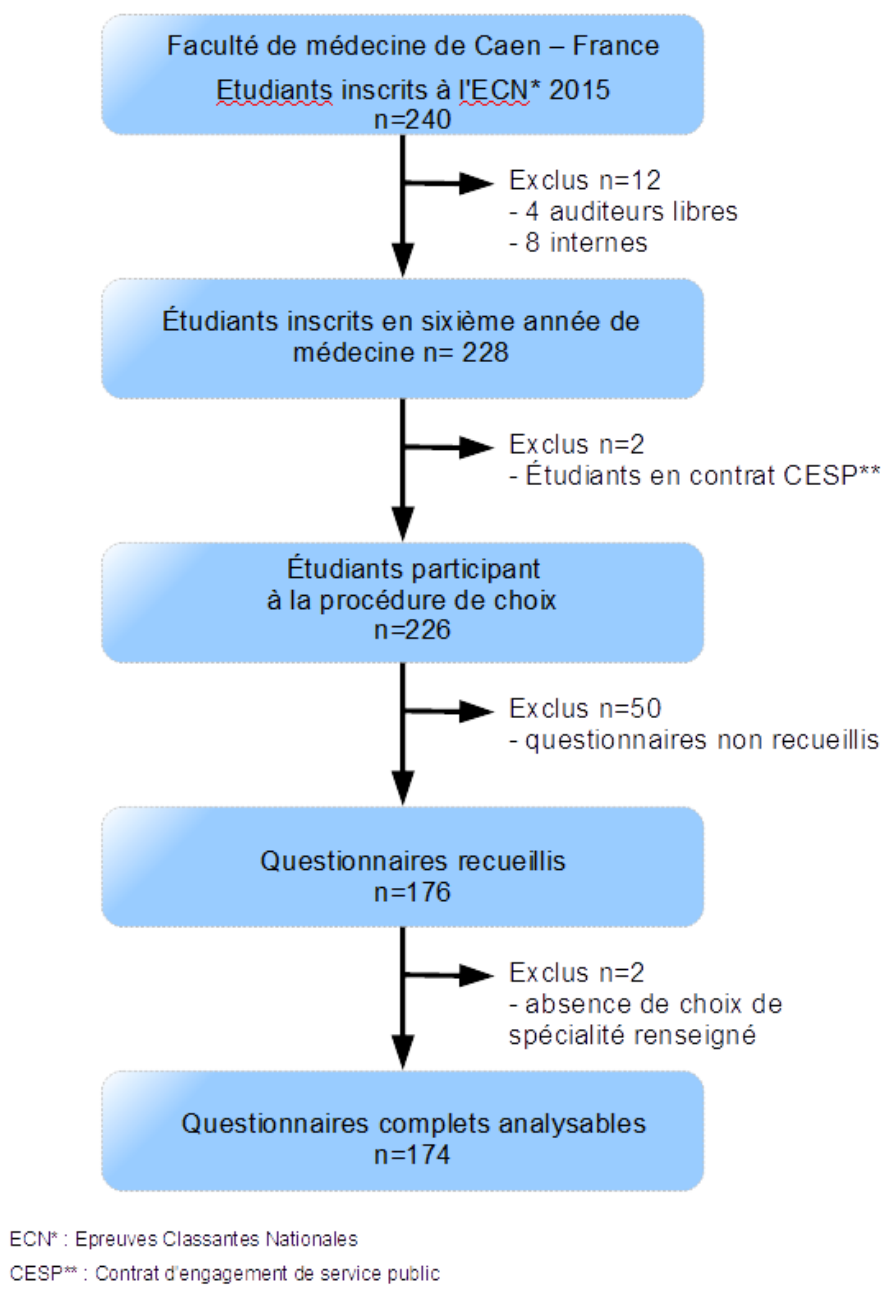

Fig. 1. Diagramme de flux du collectif de l'étude.

promotion de la santé $(p=0,036)$, la pratique en milieu rural $(p<0,001)$. La préparation aux ECN moins contraignante $(p<0,001)$, l'accès à une formation de plus courte durée $(p<0,001)$, et l'influence du conjoint $(p=0,048)$ étaient également en faveur.

Inversement, la pratique lucrative $(p=0,05)$, le prestige vis à vis des collègues $(p=0,013)$, la pratique selon la structure de soins $(p<0,001)$ et dans un grand centre urbain $(p<0,001)$, les résultats thérapeutiques immédiats $(p=0,005)$ étaient des facteurs associés au choix des autres spécialités. L'accès à une formation difficile mais stimulante $(p<0,001)$, aux dépens du choix de la ville $(p<0,001)$, étaient également en faveur.
Le regroupement statistique des informations en six classes distinctes (tableau III) retrouvait une différence en faveur du choix de la médecine générale pour les catégories «orientation sociétale » $(p=$ $0,004)$ et $«$ structures de soins $»(p=0,003)$. La classe « prestige » était en faveur du choix des autres spécialités $(p<0,001)$.

Analyse multivariée : changement de ville d'affectation

Pour les étudiants dont le premier choix était de rester dans le même centre hospitalier universitaire de formation, ce choix était associé à la possibilité de 
Tableau I. Facteurs d'influence du choix de la spécialité et coefficients de corrélation des groupes de facteurs.

\begin{tabular}{|c|c|c|c|c|c|c|c|}
\hline & & $\begin{array}{r}\text { Populatio } \\
n=1\end{array}$ & $\begin{array}{l}\text { n totale } \\
74\end{array}$ & & & & \\
\hline $\begin{array}{l}\text { Groupes } \\
\text { d'influence }\end{array}$ & Facteurs d'influence & Moyenne & $\begin{array}{l}\text { Ecart- } \\
\text { type }\end{array}$ & $\begin{array}{c}\text { Corrélation } \\
*\end{array}$ & $p$ & Moyenne & $\begin{array}{l}\text { Ecart- } \\
\text { type }\end{array}$ \\
\hline 1. Style de vie & Horaire de garde acceptable & 2,8 & 1,00 & 0,673 & $<0,001$ & 3,4 & 0,62 \\
\hline & Heures de pratique acceptables & 3,3 & 0,94 & 0,731 & $<0,001$ & & \\
\hline & Choisir ce que je veux faire en médecine & 3,6 & 0,96 & 0,558 & $<0,001$ & & \\
\hline & Faire d'autres choses en dehors de la médecine & 3,8 & 0,90 & 0,647 & $<0,001$ & & \\
\hline 2. Orientation & Se laisser des ouvertures & 3,2 & 1,19 & 0,232 & 0,002 & 3,0 & 0,72 \\
\hline & $\begin{array}{l}\text { Se concentrer sur des patients de la } \\
\text { communauté }\end{array}$ & 2,1 & 1,10 & 0,656 & $<0,001$ & & \\
\hline & Relations à long terme avec les patients & 3,6 & 1,21 & 0,692 & $<0,001$ & & \\
\hline & Guidé par l'engagement social & 3,2 & 1,20 & 0,75 & $<0,001$ & & \\
\hline & Intérêt et pratique de la promotion de la santé & 3,1 & 1,17 & 0,746 & $<0,001$ & & \\
\hline 3. Prestige & Besoin de pratique lucrative dû aux dettes & 3,6 & 1,16 & 0,51 & $<0,001$ & 3,0 & 0,74 \\
\hline & $\begin{array}{l}\text { Potentiel de revenu élevé indépendamment des } \\
\text { dettes }\end{array}$ & 3,2 & 1,05 & 0,67 & $<0,001$ & & \\
\hline & Prestige auprès des collègues & 2,3 & 1,12 & 0,675 & $<0,001$ & & \\
\hline & Activité concentrée sur les soins hospitaliers & 2,9 & 1,26 & 0,619 & $<0,001$ & & \\
\hline & Résultats immédiats des interventions & 2,9 & 1,22 & 0,698 & $<0,001$ & & \\
\hline 4. Structures de & Activité concentrée sur les soins d'urgence & 3,0 & 1,27 & 0,526 & $<0,001$ & 2,7 & 0,45 \\
\hline & $\begin{array}{l}\text { Grande diversité de problèmes et patients de } \\
\text { tous âges }\end{array}$ & 4,0 & 0,91 & 0,211 & 0,005 & & \\
\hline & $\begin{array}{l}\text { Moins grande diversité de problèmes et } \\
\text { patients d'âge précis }\end{array}$ & 1,9 & 0,94 & 0,346 & $<0,001$ & & \\
\hline & $\begin{array}{l}\text { Plus de chance de pratique dans un grand } \\
\text { centre urbain }\end{array}$ & 2,8 & 1,19 & 0,187 & 0,013 & & \\
\hline & $\begin{array}{l}\text { Plus de chance de pratique dans un milieu } \\
\text { rural }\end{array}$ & 2,3 & 1,20 & 0,462 & $<0,001$ & & \\
\hline & Formation (internat) plus court & 2,3 & 1,36 & 0,532 & $<0,001$ & & \\
\hline 5. Influence de & Formation (internat) difficile mais stimulante & 3,2 & 1,37 & 0,471 & $<0,001$ & 3,1 & 0,64 \\
\hline & $\begin{array}{l}\text { Identification à un médecin de la même } \\
\text { spécialité }\end{array}$ & 3,0 & 1,41 & 0,702 & $<0,001$ & & \\
\hline & Préparation à l'ECN moins contraignante & 2,2 & 1,26 & 0,325 & $<0,001$ & & \\
\hline & Forte influence des stages d'externat & 3,9 & 1,18 & 0,442 & $<0,001$ & & \\
\hline 6. Influence du & Privilégie le choix de la spécialité sur la ville & 4,0 & 1,31 & 0,025 & 0,741 & 3,1 & 0,69 \\
\hline & Privilégie le choix de la ville sur la spécialité & 2,2 & 1,34 & 0,003 & 0,97 & & \\
\hline & $\begin{array}{l}\text { Importance de l'avis du conjoint sur le choix } \\
\text { de ville }\end{array}$ & 4,0 & 1,11 & 0,023 & 0,764 & & \\
\hline
\end{tabular}

*Corrélation représente la corrélation des réponses entre un facteur d'influence et le groupe auquel il est associé 
Tableau II. Analyse comparative des caractéristiques sociodémographiques des étudiants selon leur premier choix de spécialité.

\begin{tabular}{|c|c|c|c|}
\hline & médecine générale & autres spécialités & valeur de $p$ \\
\hline & $n=58$ & $n=116$ & \\
\hline Moyenne d'age (écart type) & $25,1(1,20)$ & $24,89(1,47)$ & 0,038 \\
\hline Sexe féminin, $\%$ & 37,6 & 62,4 & 0,197 \\
\hline Situation, en couple, $\%$ & 37,9 & 62,1 & 0,267 \\
\hline \multicolumn{4}{|l|}{ Profession des deux parents, $\%$} \\
\hline Employé & 8,3 & 10,7 & \multirow[t]{11}{*}{0,371} \\
\hline Fonction publique (hors santé) & 6,2 & 16,6 & \\
\hline Infirmier, paramédical & 4,7 & 10,7 & \\
\hline Médecin & 4,4 & 12,4 & \\
\hline Artisan, commerçant, profession libérale & 3,6 & 5,3 & \\
\hline Pharmacien & 2,7 & 3,0 & \\
\hline Chef d'entreprise & 1,5 & 3,0 & \\
\hline Cadre & 1,5 & 2,4 & \\
\hline Agriculteur & 0,6 & 2,1 & \\
\hline Non renseigné & 0,3 & 0,0 & \\
\hline Ouvrier & 0,0 & 0,6 & \\
\hline \multicolumn{4}{|l|}{ Stage en médecine générale en 2e cycle, $\%$} \\
\hline oui & 48,3 & 51,7 & \multirow[t]{2}{*}{0,02} \\
\hline non & 24,8 & 75,2 & \\
\hline
\end{tabular}

faire autre chose en dehors de la médecine $(p=0,014)$ ou à une préférence du choix de la ville aux dépens de la spécialité $(p=0,003)$. Cette orientation résultait préférentiellement d'étudiants en couple $(p=0,005)$ mais sans influence du conjoint $(p=0,143)$. Les étudiants dont le premier choix était de partir étaient associés à un choix de formation difficile mais stimulante $(p=0,034)$, et à une préférence du choix de la spécialité sur la ville $(p<0,001)$

\section{Discussion}

Nous avons choisi d'utiliser un questionnaire dont à la fois la version princeps en anglais et la version française ont été validées en Amérique du Nord, pour rechercher une adéquation socioculturelle des facteurs d'influence et leur reproductibilité sur le modèle éducatif français. Ce questionnaire est le seul test à avoir fait l'objet d'une validation en langue française. Il est parmi les plus courts et reprend cinq dimensions distinctes connues comme influençant le choix de carrière des étudiants en médecine. Il avait démontré la meilleure valeur prédictive. D'autre part cet outil a été validé en terme de contenu (représenter toutes les thématiques souhaitables, modalités de réponse par échelle ordinale à plusieurs degrés) et de métrologie (items ordonnés, concision, redondance). L'utilisation de cet outil augmente la validité de la mesure.

Nous avons choisi d'ajouter deux classes au questionnaire, afin de prendre en compte les particularités françaises de la formation et d'affectations à l'ECN, liant la spécialité à la ville d'affectation. En effet les études ayant conçu le questionnaire 
Tableau III. Analyse comparative des facteurs d'influence du choix de la spécialité de médecine générale ou des autres spécialités.

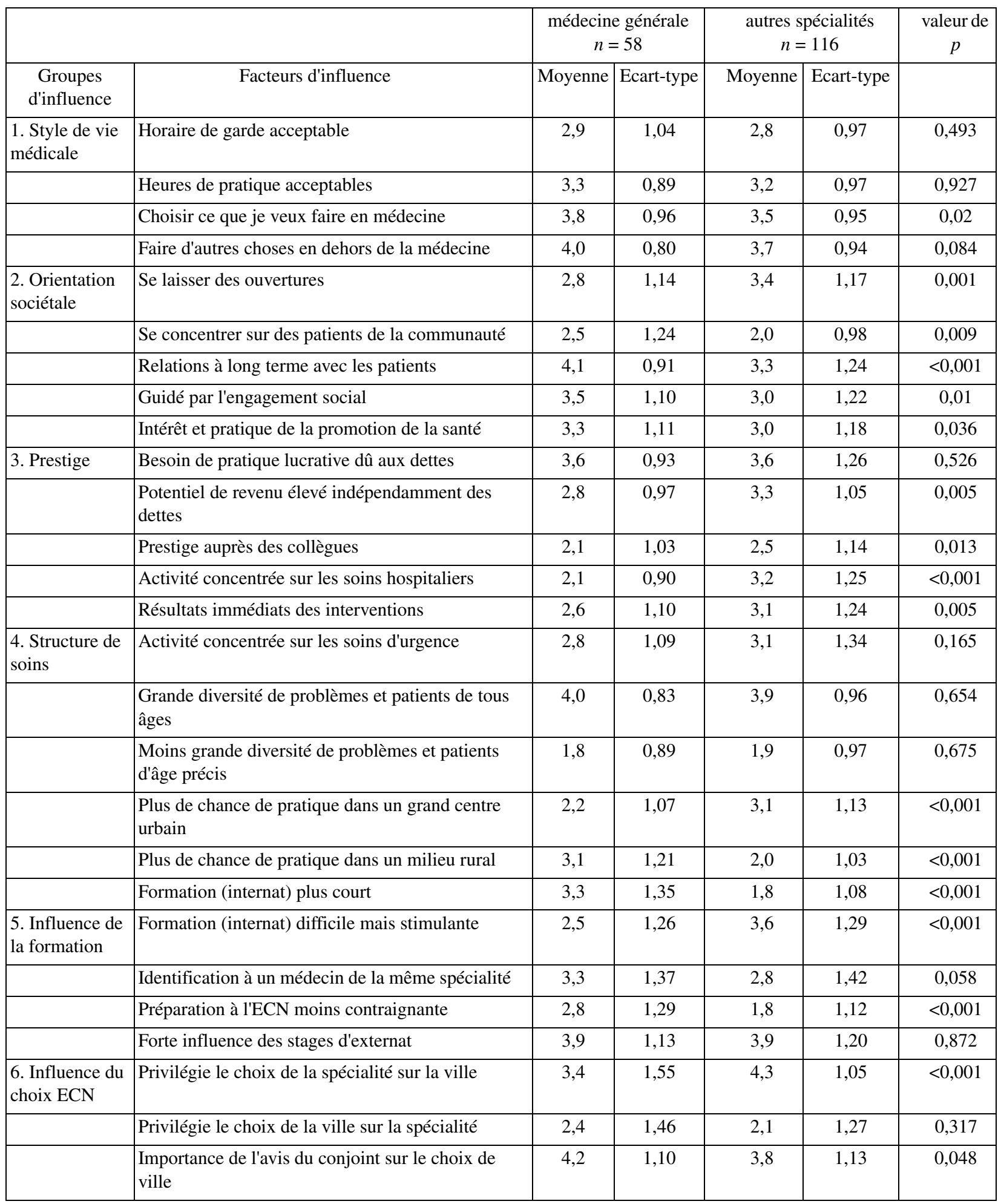


Tableau IV. Analyse comparative des groupes de facteurs d'influence du choix de la médecine générale ou des autres spécialités.

\begin{tabular}{|l|c|c|c|c|c|}
\hline & \multicolumn{2}{|c|}{$\begin{array}{c}\text { médecine générale } \\
n=58\end{array}$} & \multicolumn{2}{c|}{$\begin{array}{c}\text { autres spécialités } \\
n=116\end{array}$} & \multicolumn{2}{c|}{\begin{tabular}{c}
$p$ valeur de \\
\multicolumn{1}{|c|}{ Facteurs d'influence }
\end{tabular}} & Moyenne & Ecart-type & Moyenne & Ecart-type & \\
\hline 3. Prestige & 2,6 & 0,62 & 3,2 & 0,73 & $<0,001$ \\
\hline 4. Structures de soins & 2,9 & 0,46 & 2,6 & 0,43 & $<0,01$ \\
\hline 5. Influence de la formation & 3,1 & 0,64 & 3,0 & 0,64 & 0,27 \\
\hline 6. Influence du choix ECN & 3,0 & 0,77 & 3,1 & 0,65 & 0,81 \\
\hline
\end{tabular}

indiquaient l'intérêt de tenir compte de ces particularités dans l'utilisation du questionnaire pour des pays ayant un mode d'accès au dernier cycle des études médicales régit par concours ${ }^{[32]}$. Ces classes étaient insérées à la fin du questionnaire afin de ne pas influencer les réponses précédentes du questionnaire issu de Beaulieu et al. ${ }^{[32]}$. L'échelle de Likert à cinq classes était celle du questionnaire original et de sa version traduite. Elle permet de conserver une neutralité des questions, et un point médian évitant la tendance à l'acquiescement qui est un biais statistique.

Cette étude déclarative peut induire un biais d'information. Cependant les informations étaient recueillies directement auprès des étudiants concernés par la procédure de choix, et les réponses étaient anonymisées pour limiter ce biais de déclaration. La réalisation de l'étude à un autre moment de l'année aurait certainement apporté des résultats différents. Cependant, nous pensons qu'à ce stade (quelques semaines avant les ECN) les étudiants étaient suffisamment renseignés sur les différentes spécialités et avaient eu le temps de définir leur choix définitif de spécialité pour la suite de leur cursus.

Certains facteurs d'influence et en particulier certains freins au choix d'une spécialité ont pu ne pas être abordés, d'autant plus que le questionnaire utilisé a été élaboré et validé en Amérique du Nord. D'autres enquêtes sur les souhaits d'exercice et leurs facteurs d'influence chez des internes de médecine générale retrouvaient les mêmes facteurs d'influence en France ${ }^{[37,39]}$. Ceci pondère l'influence possible de ces facteurs non étudiés. Le questionnaire était volontairement orienté sur le choix préférentiel de l'étudiant, et non le choix réel. L'administration du questionnaire avant la tenue de l'examen classant national, et avant la connaissance du rang de classement obtenu permettait de favoriser la liberté de réponse des étudiants. Cependant on ne peut savoir s'ils ont rapporté leur préférence sans entrave ou leur choix de carrière réaliste.

Le taux de réponse était de $77 \%(n=174)$ sur notre population cible. Cependant le recueil unicentrique des données limite la validité externe des résultats.

Il existait des différences nettes dans les facteurs influençant les étudiants en médecine vers la médecine générale par rapport à la pratique d'une autre spécialité. Dans notre étude un tiers des étudiants portait un intérêt pour la médecine générale en premier choix de carrière, ce qui est comparable aux taux rapportés dans les études récentes. Trois des quatre facteurs reconnus les plus influents de notre étude sont communs aux résultats de Wright ${ }^{[34]}$.

Comme dans l'étude de Wright, et contrairement à d'autres, notre étude ne retrouvait pas d'influence du sexe de l'étudiant dans l'orientation vers la médecine générale ${ }^{[5,32-34]}$. Ceci peut être lié à l'équilibre du sexratio de notre échantillon. Une forte proportion d'étudiants avait au moins un parent exerçant dans le milieu médical $(37,8 \%)$. Certains tendent à montrer que peu de critères sociodémographiques sont significatifs sur le choix de sortie de cursus médical, indiquant que l'expérience de l'école de médecine peut avoir diminué l'influence de ces éléments ${ }^{[34]}$.

Chaque facteur était corrélé à sa classe selon Beaulieu $^{[32]}$, ce qui oriente vers l'applicabilité de ce modèle de regroupement des variables en six classes 
sur notre population. L'influence du choix aux ECN ne constitue pas un groupe homogène. Dans notre étude, le regroupement des facteurs en six classes de valeurs a mis en évidence une différence pour trois d'entre elles (orientation sociétale, prestige, structure de soins) contre cinq dans l'étude de Wright ${ }^{[34]}$. Cette différence peut découler des particularités sociologiques françaises qui modifient les qualités d'un questionnaire validé en Amérique du Nord à la fois pour sa version anglaise et pour sa version française. De même, les particularités françaises du choix d'affectation en spécialité, couplé au choix de la ville d'affectation, et dépendant du rang de classement au concours, limitent l'applicabilité externe de ces résultats.

Nous avons tenté d'analyser ces effets par la dernière classe « influence du choix aux ECN » qui retrouve une différence sur la préférence de la spécialité sur la ville entre les deux populations, qui se neutralise au regroupement des trois facteurs.

Ce regroupement des facteurs peut également induire une neutralisation des différences par des tendances opposées : la classe structure de soins regroupe des questions opposées, amenant des réponses contraires qui sont moyennées lors de l'analyse de classe. Pour tenter d'éviter cet effet nous avons inversé les résultats des questions qui comportaient leur opposé dans le même regroupement de facteurs (pratique rurale versus urbaine ; large éventail de problème versus éventail restreint). Mais cet effet a pu agir pour d'autres variables.

On peut observer que les items composant la classe « structure de soins » sont mixtes entre pratique hospitalière ou ambulatoire. La multiplication des activités partagées entre la médecine libérale et d'autres structures de soins peut expliquer que cette catégorie soit associée au choix de la médecine générale.

L'analyse des facteurs associés au changement de lieu de formation pour le dernier cycle d'études médicales retrouve l'ensemble des critères associés au choix à l'issue des ECN : les étudiants privilégiant la spécialité partent et ceux privilégiant la ville de leur choix restent, plus nettement encore s'ils sont en couple.
Nonobstant les remarques précédentes, cette étude permet de réaffirmer de nombreuses tendances connues régissant le choix de carrière des étudiants avec des données plus récentes et appliquées au modèle éducatif français, en utilisant le questionnaire de Beaulieu $^{[32]}$. La version francophone du questionnaire validé de Wright appliquée à une faculté de médecine française retrouve des résultats semblables à la population nord américaine. Ces résultats concordants montrent une certaine adéquation socioculturelle des facteurs d'influence, et leur reproductibilité sur le modèle éducatif médical francophone et français.

L'expérience d'un stage en médecine générale ambulatoire pendant le second cycle était un facteur très influent pour ce choix de spécialité, tendance déjà décrite mais non significative sous la catégorie « Role model » de l'étude de Wright ${ }^{[34,35]}$. Les étudiants intéressés par la médecine générale ont pu choisir d'effectuer ce stage ce qui peut constituer un facteur de confusion. Si tel est le cas, cette expérience semble ne pas avoir été négative et avoir conforté ces étudiants dans leur attrait pour la médecine générale au vu des résultats.

D'autres auteurs ont également démontré que l'intérêt pour la médecine générale semble augmenter à mesure que les étudiants avancent dans leur formation clinique ${ }^{[33,36]}$. Ces résultats suggèrent qu'augmenter le nombre d'expériences en médecine générale dans les années de formation clinique avant la spécialisation pourrait être bénéfique pour l'intérêt porté par les étudiants à cette spécialité. L'alternance entre stages et cours théoriques laisse actuellement peu d'espace pour ces expériences en médecine générale. Il paraitrait intéressant de multiplier ces occurrences en proposant par exemple une matinée de stage par semaine réservée à la médecine ambulatoire, tout au long du cursus.

Cette étude pourrait être approfondie en confrontant ces données avec le choix réel effectué en fonction du rang de classement aux ECN, afin de calculer l'indice d'espérance atteinte (taux de choix espérés accessibles) et ainsi de confronter choix espéré et choix réel. Par ailleurs, elle pourrait être poursuivie en recrutant une nouvelle promotion 
d'étudiants accédant au choix de spécialité. L'objectif serait de comparer les différences dans les facteurs d'influence et dans les choix de spécialité chez ces étudiants ayant bénéficié de la réorganisation de l'enseignement des études médicales depuis le début de leur cursus.

\section{Conclusion}

Les étudiants en médecine qui préfèrent la médecine générale comme choix de carrière semblent être influencés par un ensemble de facteurs différents de ceux qui préfèrent les carrières de spécialité. Une préférence pour la médecine générale est associée à trois groupes de valeurs : l'orientation sociétale, le choix de la structure de soins et le prestige de la spécialité. Par ailleurs, le fait d'avoir effectué un stage en médecine générale pendant la première partie du cursus est associé au choix ultérieur de cette spécialité. A l'heure où l'évolution de la démographie médicale tend à faire exercer une pression sur l'orientation des choix de spécialisation, ces résultats suggèrent que l'on pourrait s'orienter vers de nouvelles stratégies pour favoriser l'attrait des étudiants vers la médecine générale, notamment en renforçant les expériences en médecine ambulatoire tout au long du cursus de formation.

\section{Contributions}

Antoine Aubrion a participé à la conception du protocole, au recueil des données, à l'interprétation des résultats et à l'écriture du manuscrit. Pascal Goncalvès a participé à l'interprétation des résultats et à l'écriture du manuscrit. Vincent Kolwaski a participé à l'analyse statistique. Anaïs Reichling a participé à la relecture du manuscrit.

\section{Approbation éthique}

Non sollicitée

\section{Déclaration d'intérêts}

Aucun des auteurs ne déclare de conflit d'intérêts en lien avec le contenu de cet article.

\section{Références}

1. National Residency Matching Service (NRMP). Washington (DC). [On-line] Disponible sur : www.nrmp.org

2. Observatoire national de la démographie des professions de santé. Rapport annuel 2006-2007, Tome 1 : La médecine générale. Paris : ONDPS, 2008.

3. Brotherton S, Rockey PH, Etzel S. US graduate education, 2004-2005: trends in primary care specialties. JAMA 2005;294:1075-82.

4. Cooper RA. Weighing the evidence for expanding physician supply. Ann Intern Med 2004;141:705-14.

5. Attal-Toubert K, Vanderschelden M. La démographie médicale à l'horizon 2030 : de nouvelles projections nationales et régionales détaillées. DREES - Solidarité et santé -n¹2 2009

6. Bowler I, Jackson N. Experiences and career intentions of general practice registrars in Thames deaneries : postal survey. BMJ 2002;324:464-5.

7. Schweyer FX, Levasseur G. Profil et devenir des jeunes médecins généralistes en Bretagne. État des connaissances à partir d'une étude documentaire et d'une revue de la littérature. Paris : URML Bretagne (éditeur), 2003.

8. Sicart D. Projection médecins 2002-2020. DREES, Documents de travail, Série Statistiques. 2002:30.

9. Yvon B, Lehr-Drylewicz AM, Bertrand P, Féminisation de la médecine générale : faits et implications, une enquête qualitative en Indre et Loire. Médecine : de la médecine factuelle a nos pratiques 2007/02;3: 83-8.

10. Benson JA Jr, Kimball HR. Where have all the primary care applicants gone ? N Engl J Med 1992;326:177880.

11. Fincher RM. The road less traveled-attracting students to primary care. N Engl J Med 2004;351:630-2.

12. Soulier E, Grenier C, Lewkowicz M. La crise du médecin généraliste : une approche cognitive de la profession. Revue Médicale de l'Assurance Maladie 2006;37:99-108. 
13. Sarma S, Thind A, Chu MK. Do new cohorts of family physicians work less compared to their older predecessors? The evidence from Canada. Soc Sci Med 2011;72:2049-58.

14. Canadian Resident Matching Service. Reports and statistics, 2009. $R-1$ match reports. Ottawa, ON: Canadian Resident Matching Service, 2009.

15. Lefevre JH, Roupret M, Kerneis S, Karila L. Career choices of medical students: a national survey of 1780 students. Med Educ 2010;44:603-12

16. Décret $n^{\circ} 2004-67$ du 16 janvier 2004 relatif à l'organisation du troisième cycle des études médicales. Journal officiel de la République française $\mathrm{n}^{\circ} 15 \mathrm{du}$ 18 janvier 2004: 1394; texte ${ }^{\circ} 14$

17. Arrêté du 29 janvier 2004 relatif à l'organisation des épreuves classantes nationales anonymes donnant accès au troisième cycle spécialisé des études médicales. Journal officiel de la République française $\mathrm{n}^{\circ} 30$ du 5 février 2004: 2519; texte ${ }^{\circ} 36$

18. Bachelet M, Les affectations des étudiants en médecine à l'issue des épreuves classantes nationales en 2013.DRESS, Etudes et résultats $2014 ; 894$

19. Bismuth M, Rougé ME, Poutrain JC, Bismuth P, Boyer P, Escourrou B et al. Pourquoi les internes choisissent-ils la médecine générale? La médecine générale revient dans les premiers choix... Médecine 2009;9:136-41

20. Hernandez E. ISNAR-IMG. Enquête nationale sur la formation des internes de médecine générale. L'antidote 2014(3):6-7;

21. Association of American Medical Colleges (AAMC). Recent studies and reports on physician shortages in the US. Washington, DC: AAMC. 2011 [On-line] Disponible sur : https://www.aamc.org/download/ 100598/data/recentworkforcestudiesnov09. pdf.

22. Colwill JM, Cultice JM, Kruse RL. Will generalist physician supply meet demands of an increasing and aging population? Health Aff 2008;27:w232-41.

23. Reed VA, Jernstedt GC. Understanding and improving medical student specialty choice: a synthesis of the literature using decision theory as a referent. Teach Learn Med 2001;13:117-29.

24. Senf JH, Campos-Outcalt D, Kutob R. Factors related to the choice of family medecine:a reassessment and literature review. J Am Board Fam Pract 2003;16: $502-12$
25. Lambert T, Goldacre R, Smith F, Goldacre MJ. .Reasons why doctors choose or reject careers in general practice: national surveys. Br J Gen Pract 2012;62: e851-e858.

26. Lydon S, O'Connor P, McVeigh T, Offiah C, Byrne D.Medical speciality choice: does personality matter? Ir Med J 2015;108:75-8.

27. Nabli Ajmi T, Bougmiza MI, Mtiraoui A. Attitudes des étudiants tunisiens en médecine vis-à-vis de la pratique médicale : différences en fonction du sexe. East Mediterr Health J 2008;14:686-96.

28. Owen JA, Hayden GF, Connors AF Jr. Can medical school admission committee members predict which applicants will choose primary care careers? Acad Med 2002;77:344-9.

29. Mobilos S, Chan M, Brown JB. Women in medicine: the challenge of finding balance. Can Fam Physician 2008;54:1285-86.e5.

30. Murdoch MM, Kressin N, Fortier L, Giuffre PA, Oswald L. Evaluating the psychometric properties of a scale to measure medical students' career-related values. Acad Med 2001;76:157-65

31. Wright B, Scott I,Woloschuk W, Brenneis F. Career choice of new medical students at three Canadian universities: family medicine versus specialty medicine. CMAJ 2004;170:1920-4.

32. Beaulieu MD, Haggerty J, Bouharaoui F, Goulet F, Validité et fidélité de la version française d'un questionnaire portant sur le choix de carrière des étudiants en médecine. Pédagogie Médicale 2010;11;7-17

33. Gill H, McLeod S, Duerksen K, Szafran O. Factors influencing medical students' choice of family medicine: Effects of rural versus urban background. Canadian Family Physician. 2012;58:e649-e657.

34. Scott I, Gowans M, Wright B, Brenneis F, Banner S, Boone J. Determinants of choosing a career in family medicine. CMAJ 2011;183:E1-8

35. Hauer KE, Durning SJ, Kernan WN, et al. Factors associated with medical students' career choices regarding internal medicine. JAMA 2008;300:115464

36. Scott I1, Gowans M, Wright B, Brenneis F.Stability of medical student career interest: a prospective study. Acad Med 2012;87:1260-7. 
37. Septier-Guelff J, Fanello S, Connan L, Paré F, Bouton C.Angers general practice residents' career projects in 2012. Santé Publique 2014;26:65-74.

38. Rouger AS, Ladner J, La médecine générale : Un choix par défaut aux Epreuves Classantes Nationales? : Étude multicentrique sur la concrétisation des souhaits des étudiants aux ECN. Thèse pour le Diplôme d'état de doctorat en médecine. Faculté de Rouen, 2013
39. Gaidioz C, Ruhlmann S. Pourquoi la specialite medecine générale est-elle mal classée aux choix des épreuves classantes nationales ? Etude des représentations des étudiants lyonnais du PCEM 1 au DCEM 4. Thèse pour le Diplôme d'état de doctorat en médecine. Faculté de Rouen, 2008

Correspondance et offprints: Antoine AUBRION, Hopital Robert Bisson, 4 rue Roger Aini, 14100 Lisieux, France. Téléphone : (+33) 0604187275.

Mailto : aubrion.antoine@gmail.com 
Annexe. Questionnaire de Beaulieu ${ }^{[32]}$ portant sur le choix de carrière des étudiants en médecine.

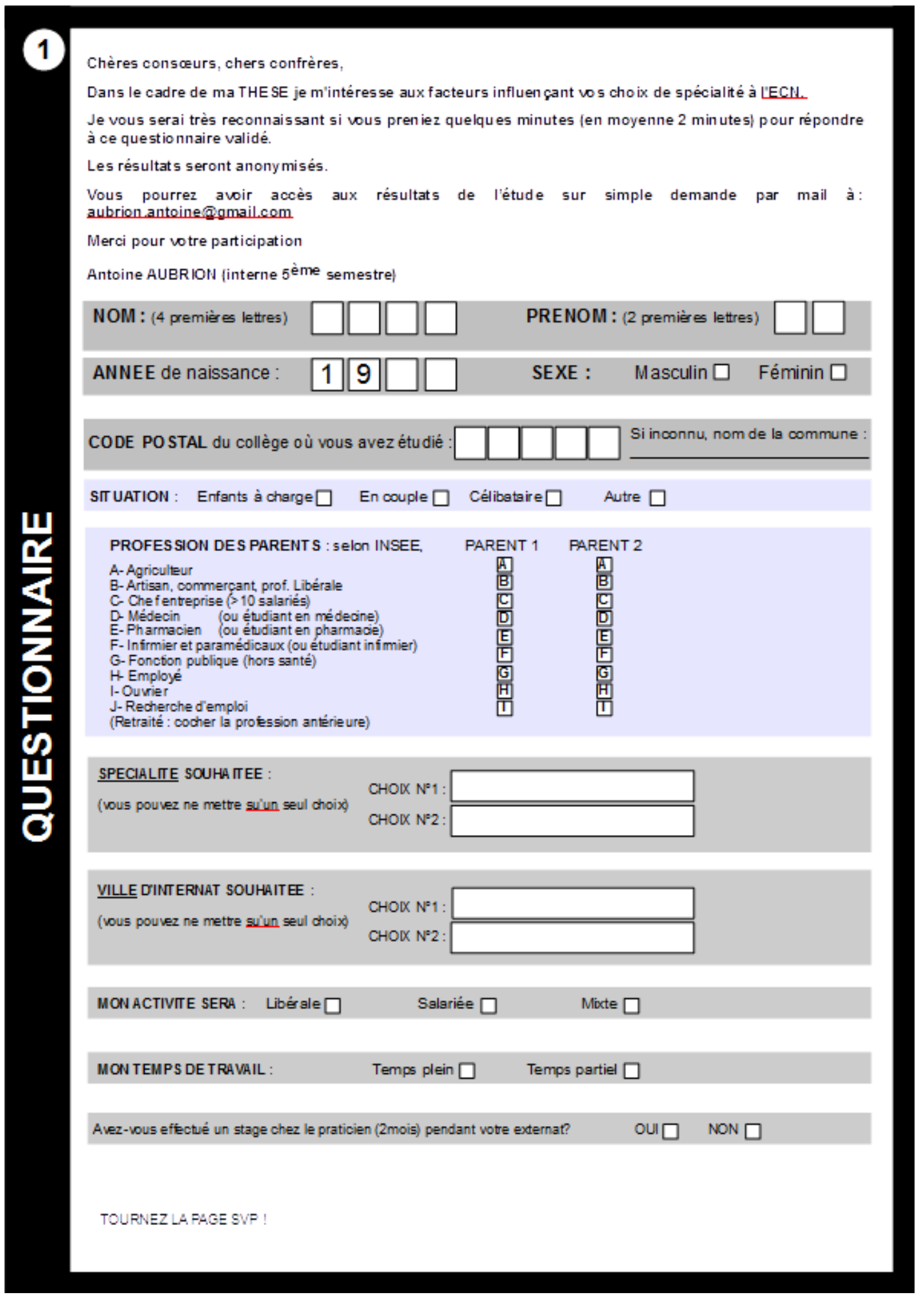




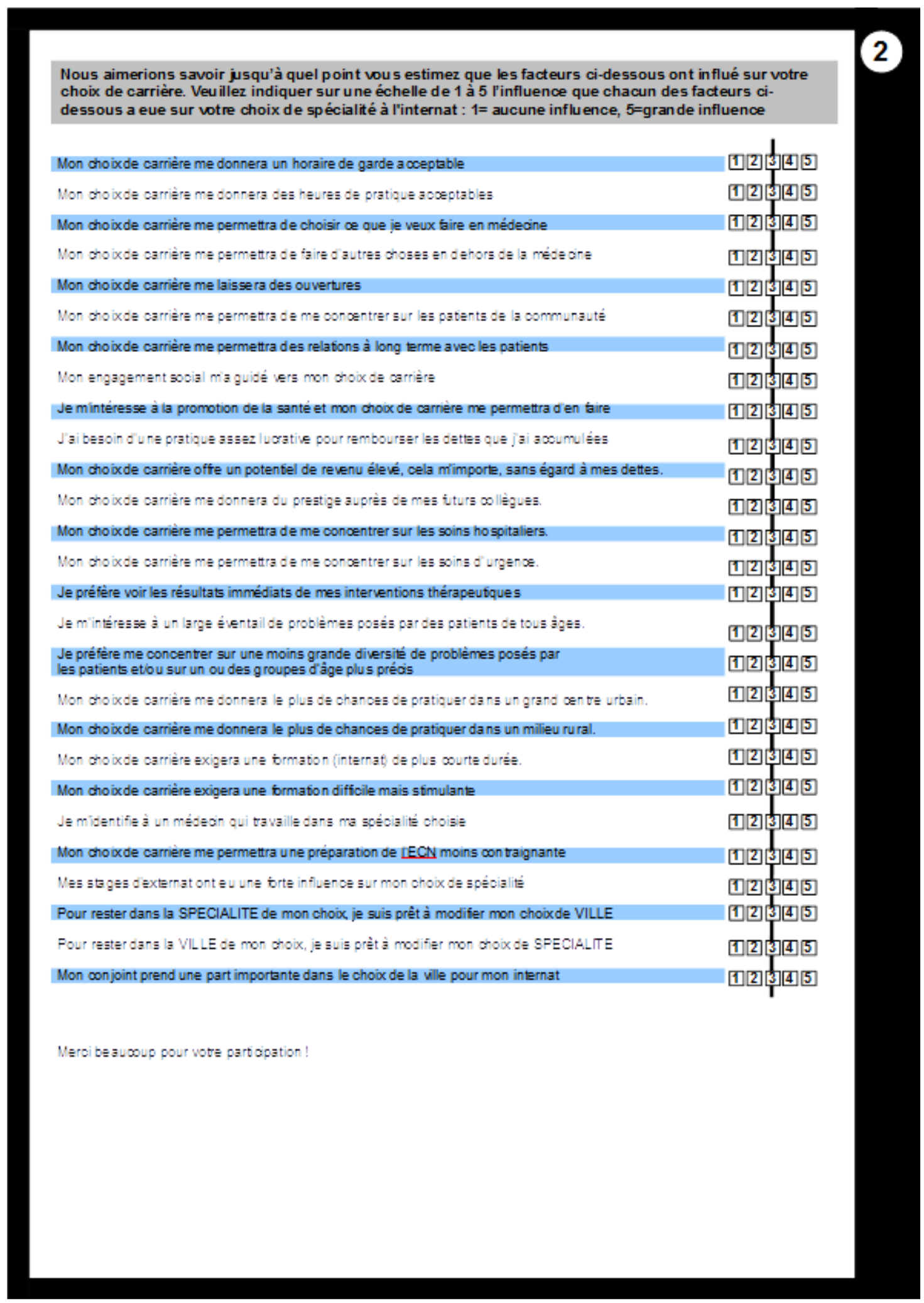

